\title{
La cobertura de la Cuestión Malvinas en los medios de comunicación gráfica (2011-2015)
}

\author{
Lilian Berardi* \\ Oriana Cherini** \\ Paola Zárate ${ }^{* * *}$
}

\section{Resumen}

El objetivo del presente trabajo es identificar y analizar la cobertura periodística de la cuestión Malvinas durante el segundo mandato presidencial de Cristina Fernández (2011-2015) en los principales medios gráficos de nuestro país: La Nación, Página/12, Clarín y los Comunicados de Prensa de la Cancillería, en función a los lineamientos de la Política Exterior Argentina (PEA). Al mismo tiempo se apunta a identificar los principales los actores y temas vinculados a esta cuestión. El planteo de esta problemática se vincula al rol cada vez más relevante que los medios de comunicación adquirieren en lo que la denominada "imposición de la visión legítima”.

Palabras claves: Cuestión Malvinas - cobertura periodística - lineamientos - Política Exterior Argentina (PEA) - Cristina Fernández

\section{The journalistic coverage of the Malvinas issue in the graphic media (2011-2015)}

\begin{abstract}
Summary
The objective of this article is to analyze the journalistic coverage of the Malvinas issue during the second presidential term of Cristina Fernández (2011-2015) in the main graphic media of our country: La Nación, Page/12, Clarín and the Press Releases of the Ministery or Foreign Relations, according to the guidelines of the Argentine Foreign Policy. At the same time we want to identify the main actors and issues linked to this issue. The way this problem is featured is linked to the increasingly important role that the media acquire in what is known as the "imposition of the legitimate vision".
\end{abstract}

Keywords: Falklands Question - journalistic coverage - guidelines - Argentine Foreign Policy - Cristina Fernández

TRABAJO RECIBIDO: 25/03/2019 TRABAJO ACEPTADO: 02/05/2019

\footnotetext{
* Licenciada en Relaciones Internacionales, Universidad Nacional de Rosario (UNR, Argentina). Miembro del Observatorio de Política Exterior Argentina de la UNR. Correo electrónico: liliannoeliab@hotmail.com

** Licenciada en Relaciones Internacionales, Universidad Católica Argentina. Miembro del Observatorio de Política Exterior Argentina de la UNR. Correo electrónico: oricherini@ gmail.com

*** Estudiante avanzada de la Licenciatura en Relaciones Internacionales, Universidad Nacional de Rosario (UNR, Argentina). Miembro del Observatorio de Política Exterior Argentina de la UNR. Correo electrónico: paoz96@hotmail.com
} 


\section{Introducción}

Desde hace ya más de dos siglos uno de los temas significativos de la Política Exterior Argentina (PEA) es la Cuestión Malvinas. De allí que se constituye en un tema constante y relevante de la agenda de política exterior (PE) en los distintos gobiernos, más allá de su signo político. Ello se ha visto reforzado luego de la Guerra de Malvinas y del retorno a la democracia en el año 1983, pudiéndose observar que cada una de las presidencias ha fortalecido dicha temática como uno de sus principales objetivos, aunque con matices propios.

Es menester indicar que la cuestión Malvinas hace referencia a una disputa entre la República Argentina y el Reino Unido, que comprende la soberanía territorial sobre las Islas Malvinas, Georgias del Sur, Sandwich del Sur y los espacios marítimos circundantes, más el lecho y el subsuelo. Esta controversia es de carácter bilateral y desde las Naciones Unidas se insta a las Partes a resolverla en forma pacífica, contemplando los intereses de los habitantes de las islas (Resolución 2065 de la Asamblea General de Naciones Unidas).

El punto clave del conflicto post-guerra es el reclamo de soberanía sobre las Islas Malvinas e Islas del Atlántico Sur, que constituye para la Argentina un objetivo permanente e irrenunciable, tal como lo establece la Constitución Nacional, y que además representa un tema de gran sensibilidad en nuestra sociedad. Sin embargo, esta disputa no afecta únicamente la cuestión soberana, sino también conflictos económicos, humanitarios, militares y de seguridad.

Dentro de estas temáticas, cabe subrayar que el área bajo disputa despierta los intereses económicos de varios actores, entre los cuales se encuentra principalmente el sector privado. En este sentido, se observa la presencia cada vez mayor de empresas hidrocarburíferas extranjeras en las aguas aledañas a las islas, con expectativas de hallar hidrocarburos comercialmente viables. Y a su vez, se encuentran las compañías pesqueras foráneas que explotan los recursos ictícolas, como consecuencia de la venta unilateral de las licencias por parte del Reino Unido. Señalamos que dicha actividad económica constituye una de las principales fuentes de ingresos de los habitantes de las islas. En relación a esto, durante el gobierno de Cristina Fernández se aprobaron distintas normativas legales para intentar limitar el accionar de dichos actores, tanto públicos como privados.

También en lo relativo a las cuestiones de carácter humanitario, se puede hacer referencia a la cooperación bilateral entre Argentina y Reino Unido para posibilitar la visita de los familiares de los ex combatientes al cementerio Darwin en las Islas Malvinas, y para llevar adelante el proceso de identificación de los soldados que yacen en dicho cementerio.

Otro punto relevante a considerar en la Cuestión Malvinas es el creciente proceso de militarización de la base Monte Agradable situada en la Isla Soledad, promovido por el gobierno inglés. Su incremento es notorio finalizado el conflicto de 1982 y actualmente, representa una amenaza a la seguridad de la región.

Al mismo tiempo, en cuanto al apoyo regional e internacional, es relevante destacar los numerosos respaldos que recibió Argentina por parte diversos países del mundo, y también, de organizaciones multilaterales de América Latina y del Caribe, incluyendo las Cumbres Iberoamericanas y Sudamericanas, Mercado Común del Sur (MERCOSUR), Asociación Latinoamericana de Integración (ALADI), Unión de Naciones Suramericanas (UNASUR), Sistema de Integración Centroamericano (SICA), y Comunidad de Estados Latinoamericanos y Caribeños (CELAC).

Es por lo anteriormente descripto y sumado a otros aspectos que exceden el plano político y económico, que lo comunicacional posee una importancia fundamental en la agenda de la PEA, dentro de un mundo globalizado donde la información fluye y se reproduce a través de distintos medios, entre ellos los periodísticos, en una sociedad internacional que exige actualidad y renovación de la información. 
En tal sentido se plantea el siguiente interrogante: ¿Cómo fue la cobertura periodística de la Cuestión Malvinas en los principales medios gráficos argentinos con referencia a los lineamientos de PEA del período 2011-2015?

En el marco de un proyecto de investigación más amplio ${ }^{1}$ este trabajo se propone como objetivo general analizar la cobertura periodística de la cuestión Malvinas en los principales medios gráficos de nuestro país durante el segundo período presidencial de Cristina Fernández (2011-2015), relacionado con los lineamientos de la PEA en dicho período. Y, de modo específico, identificar los principales actores y temas vinculados a la Cuestión Malvinas e indagar sobre la relación entre ellos.

El planteo de esta problemática se relaciona al rol cada vez más relevante que los medios de comunicación están adquiriendo, en lo que es la "imposición de la visión legítima" (Lucero, 2006:208). Es decir, la información que llega a los ciudadanos respecto a la Cuestión Malvinas, proviene de un recorte tanto ideológico como técnico de los medios de difusión (Lucero, 2006), lo que lleva a querer descifrar cómo es dicho recorte, y cuál es la información que llega a la ciudadanía.

Asimismo, los medios cumplen un rol importante como actor internacional no estatal, dentro de un sistema internacional cada vez más heterogéneo, como comunicadores que influyen en establecer los temas de agenda, y qué información de dichos temas se comunican. Una de las teorías que estudia este fenómeno es la agenda setting, que plantea: (...) cómo los medios ejercen influencia en las audiencias mediante los temas considerados de mayor relevancia. El medio no decide por el público qué es lo que éste tiene que pensar u opinar sobre un hecho, aunque sí decida cuáles son las cuestiones que van a estar en el candelero o en la opinión pública. A este conjunto de contenidos se le denominará: la agenda (...) (Raquel Rodriguez Diaz, 2004:15).

A los fines de este trabajo se consideró a la PE como aquella área de actividad gubernamental que es concebida como las relaciones entre el Estado y otros actores, particularmente otros Estados (pero no exclusivamente) en el sistema internacional (...) (Lasagna, 1995:389).

Para complementar esta concepción se incorporan los aportes de Russel, al definir a la PE como el área particular de acción política gubernamental que abarca tres dimensiones analíticamente separables -político-diplomática, estratégico-militar y económica- y que se proyecta al ámbito externo frente a una amplia gama de actores e instituciones gubernamentales y no gubernamentales, tanto en el plano bilateral como multilateral (...) (Russell, 1990:255).

También el tratamiento periodístico de los hechos de la PE establece vínculos con los contextos internos y externos y con las agendas temáticas de la política nacional y mundial. En este sentido, el modo en que los medios abordan la Cuestión Malvinas, establece prioridades en la política editorial en torno a la agenda política argentina (Lucero, 2006).

La importancia de utilizar como recurso informativo los periódicos resulta de la relevancia que han adquirido los medios de comunicación para establecer la agenda y difundir el recorte de la información que establezcan. En el área de las Relaciones Internacionales, esto ha impactado en la comunicación de los distintos órganos o áreas de la PE de los Estados, en la difusión de los temas de agenda (Castillo, 2015:13).

En función de los objetivos descriptos, este trabajo sostiene la siguiente hipótesis: la cuestión de fondo relativa a la soberanía por las Islas Malvinas fue la que tuvo la mayor frecuencia de aparición en los medios gráficos, en las noticias ligadas a ésta temática. Esta

\footnotetext{
1 Proyecto de Investigación y Desarrollo (PID), Universidad Nacional de Rosario titulado "La construcción de representaciones mediáticas de la Política Exterior Argentina a través de los medios de comunicación gráfica: el Observatorio de Política Exterior Argentina 2011-2017”, código 1POL275, Universidad Nacional de Rosario.
} 
frecuencia se vincula a la relevancia del tópico en la PEA, asociado, en mayor medida, a los actores públicos en la etapa analizada. Esto último permite inferir que el reclamo de soberanía corresponde principalmente a los actores públicos, en particular los Estados nacionales y sus representantes oficiales.

Este artículo se estructura en tres secciones adicionales a esta introducción. La primera de ellas describe los lineamientos de la PEA usando bibliografía especializada como fuente principal; la segunda parte evalúa la cobertura periodística de la Cuestión Malvinas en el período de estudio a partir de las frecuencias de aparición del título, como la identificación de actores y temas ligados a dicho título. Finalmente, se presentan algunas conclusiones preliminares que operan como un primer insumo de la labor grupal realizada en el marco del PID.

\section{Lineamientos de la PEA y de la PE hacia la Cuestión Malvinas}

Se enmarca la cuestión bajo análisis tomando en cuenta que el Ministerio de Relaciones Exteriores y Culto de la Nación considera a la PEA como: “(...) una herramienta esencial para preservar, defender y resguardar la soberanía, dignidad e intereses de la República en el ámbito continental y mundial, así como también para contribuir a la expansión, consolidación y difusión de un enfoque de desarrollo económico y social que persigue la mejora de la calidad de vida de sus ciudadanos, el avance de la justicia social y la disminución de la pobreza, de modo de garantizar la alineación y consistencia entre la política exterior y el modelo de desarrollo nacional con inclusión social".

Acorde con el recorte temporal realizado y que abarca la segunda presidencia de Cristina Fernández (2011-2015) debe señalarse que se mantuvieron los principales lineamientos de PE implementados por su antecesor, Néstor Kirchner, aunque su gestión generó rasgos particulares. En tal sentido, el gobierno de Fernández se caracterizó por matices acordes a los condicionantes tanto internos como externos, a la vez que los lineamientos generales siguieron intactos en cuanto a su contenido sustancial.

Con referencia al período, Corigliano sostiene que se puede entender a través de la yuxtaposición de dos variables realistas y dos idealistas. Dentro del realismo geopolítico: la variante ingenua y la variante pragmática. La primera hace referencia a entender a la autonomía como la libertad de acción de un Estado en relación a otros, mientras que la segunda apunta a convertir a la Argentina en un socio confiable para la alianza occidental en los temas de terrorismo y narcotráfico. En segundo lugar, desde el idealismo, la variable wilsonianaperiférica y la grociana-periférica. La wilsoniana-periférica hace referencia a juzgar las violaciones de derechos humanos ocurridos en la Argentina, y la grociana-periférica, en confiar en las Naciones Unidas como un canal para reclamar cuestiones referentes a la AMIA, Malvinas, etc. (Corigliano, 2006).

Por lo tanto se puede plantear que la PE de Fernández ha tenido un perfil autonómico, caracterizado por dos esferas de acción: los problemas financieros, comerciales y de inversión con Estados Unidos y con China; y la relación con los países latinoamericanos, en la búsqueda de la defensa de la democracia, la resolución de conflictos intrarregionales, el fomento de la integración (Busso, 2014:29). Los principales temas de PE durante el gobierno de Cristina Fernández fueron: Derechos Humanos, Desarrollo Tecnológico, Deuda Externa, Espacios Multilaterales, Vínculos con América Latina, Jerarquización de Relaciones con China y Rusia, entre otros (Busso, 2014).

\section{La PE hacia la Cuestión Malvinas}

La guerra de Malvinas fue un evento trágico para el pueblo argentino y determinante para la PEA posteriormente. Luego de 1982, todos los gobiernos de la República Argentina, en mayor o menos medida, trataron como tema de agenda a la Cuestión Malvinas. 
Durante la administración de Cristina Fernández se observó una postura más dura respecto a las medidas adoptadas hacia el Reino Unido en relación al gobierno de Néstor Kirchner. Asimismo, se concuerda con Bologna (2014), al considerar que las políticas exteriores de los gobiernos de Cristina Fernández y Néstor Kirchner, mantuvieron una continuidad en cuanto a los temas vinculados a Malvinas.

En concordancia con esto, se destaca el discurso de toma de posesión como presidenta de la Nación, en el que calificó como "irrenunciables e indeclinable"2 el reclamo de soberanía sobre las islas por parte de la República Argentina (Fernández, 2007).

La Cuestión Malvinas, como plantea Simonoff (2009), fue uno de los temas de agenda más destacados de la PEA. Así lo sostiene el Ministerio de Relaciones Exteriores y Culto de la Nación, al afirmar que los lineamientos de la PEA en referencia a la Cuestión Malvinas en este período buscaron: “(...) Procurar la recuperación del ejercicio de soberanía sobre los mencionados archipiélagos, conforme al derecho internacional y de acuerdo con el mandato de la Disposición Transitoria Primera de la Constitución Nacional; difundir en la comunidad internacional los derechos de soberanía de la República Argentina sobre las Islas Malvinas, Georgias del Sur y Sandwich del Sur y los espacios marítimos circundantes; procurar el cumplimiento del mandato de las Naciones Unidas de reanudar las negociaciones de soberanía con el Reino Unido de Gran Bretaña e Irlanda del Norte relativas a la Cuestión de las Islas Malvinas; consolidar y ampliar el respaldo de la comunidad internacional a la posición argentina en los foros internacionales, regionales y birregionales; y promover en el exterior, en ámbitos periodísticos, parlamentarios, culturales, mediáticos, el respaldo y solidaridad con la posición argentina en la Cuestión Malvinas (...)”3.

Asimismo, cabe mencionar, como dato muy relevante, que durante esta administración se creó, en el Ministerio de Relaciones Exteriores y Culto de la Nación, la Secretaría de Asuntos relativos a la Islas Malvinas, Georgias del Sur, Sandwich del Sur y los espacios marítimos circundantes en el Atlántico Sur, con el fin de potenciar las acciones nacionales relativas a la defensa de los legítimos derechos de soberanía (Bologna, 2014:44).

Al mismo tiempo puede señalarse que en la misma línea que su antecesor, Fernández desarrolló una estrategia que buscó el apoyo de la comunidad internacional colocando la Cuestión Malvinas en todos los foros y organismos internacionales (OI) de que Argentina formaba parte. Entre ellos la UNASUR, cuyos países miembros respaldaron la posición argentina a través de diversas declaraciones, en la disputa de soberanía con el Reino Unido referida a la Cuestión Malvinas. En dichos documentos se instó a las partes a reanudar las negociaciones diplomáticas. Es así que en la Declaración del año 2010 rechazaron las actividades petroleras realizadas en la Plataforma Continental argentina por el Reino Unido, ya que se oponen a las Resolución 31/49 de la Asamblea General de las Naciones Unidas.

En este sentido, todos los países de América Latina se han pronunciado dando su respaldo a los derechos de Argentina en la disputa de soberanía. Lo han hecho reiteradamente y en múltiples foros tales como el MERCOSUR, la UNASUR, el Alianza Bolivariana para los Pueblos de Nuestra América (ALBA), y, también junto a los países del Caribe, en el seno de la CELAC.

Del mismo modo Fernández logró cosechar apoyos de los socios extra-bloque como es el caso de las 54 naciones del África, que respaldaron los derechos argentinos en la disputa de soberanía en el marco de las Cumbres de Países Sudamericanos y Africanos (ASA). También Rusia, China, India y los países árabes manifestaron su respaldo. Con ello se buscó transformar

\footnotetext{
${ }^{2}$ Discurso de toma de posesión de Cristina Fernández ante el Congreso Nacional, el 10 de diciembre de 2007.

${ }^{3}$ Disponible en http://concursos2015.cancilleria.gob.ar/userfiles/Lineamientos-politica-exterior-20132015.pdf.
} 
el reclamo argentino sobre Malvinas no sólo como causa de interés regional sino también internacional.

Además es necesario recordar que las Naciones Unidas, la Organización de Estados Americanos (OEA), las Cumbres Iberoamericanas, la Zona de Paz y Cooperación del Atlántico Sur (ZPCAS), el Grupo de los 77 más China y el Sistema de Integración Centroamericano (SICA), se han manifestado reiteradamente a favor de la reanudación de las negociaciones bilaterales entre Argentina y Reino Unido. Como sostiene Bologna, la estrategia seguida por Argentina en diferentes ocasiones de presionar internacionalmente al Reino Unido en los organismos multilaterales ha permitido que el gobierno nacional obtenga apoyos internacionales a sus reclamos sobre las Malvinas, aunque la cuestión de fondo, que es el tema de la soberanía, continúa sin discutirse (Bologna, 2010:261).

Si se recorre la trayectoria desde 1982, la PEA relativa a la Cuestión Malvinas tuvo dos objetivos principales: en primer lugar, instaurar y defender la postura del gobierno argentino en torno a la recuperación de la soberanía sobre dichos territorios y denunciar los actos unilaterales por parte del gobierno británico en temas asociados a la soberanía sobre Malvinas, esto es, la militarización del Atlántico Sur y la explotación de los recursos ictícolas y recursos hidrocarburíferos, entre otros.

Durante la presidencia de Fernández se aprobaron algunas medidas legales tendientes a frenar las acciones unilaterales inglesas respecto a impulsar la exploración y explotación de los recursos hidrocarburíferos hallados en la plataforma argentina. El Reino Unido concedió licencias para desarrollar dichas actividades económicas a empresas extranjeras sin el consentimiento del gobierno argentino. En este sentido la administración Fernández modificó en el año 2013- la Ley Nacional $\mathrm{N}^{\circ} 26.659^{4}$ (2010), que establece las condiciones para la exploración y explotación de hidrocarburos en la plataforma continental argentina, que además inhabilita a toda persona física o jurídica a realizar actividades en Argentina, y establece sanciones de entre 5 y 20 años para los infractores. También modificó la Resolución $\mathrm{N}^{\circ} 407 / 2007^{5}$, mediante la cual no sólo prohíbe operar en la Argentina a aquellas empresas que tuvieran participación o vinculación con compañías que realizaran actividades de exploración y explotación de hidrocarburos en la plataforma continental argentina sin permiso de la autoridad, sino que, en el caso de aquellas empresas que en el pasado hubieran mantenido algún tipo de vinculación con sociedades involucradas en dichas actividades ilícitas, deberán informar a la autoridad competente, con carácter de declaración jurada, la inexistencia actual de tales vínculos y su voluntad de sujetarse a las disposiciones de la resolución.

En el período bajo estudio los temas relacionados a la soberanía sobre las Islas Malvinas, tales como la exploración y explotación de recursos hidrocarburíferos, recursos ictícolas y la creciente militarización de la base en Monte Agradable, tuvieron un papel destacado, ya que fueron motivo de rispideces con el gobierno inglés. Si se sigue a Bologna (2006) en la diferenciación de agendas que el autor plantea, se desprende la necesidad de analizar la agenda económica, que incluye actividades referidas - puntualmente- a la exploración y explotación de los hidrocarburos en la plataforma continental argentina por parte de las empresas privadas, y la explotación ictícola en las aguas bajo disputa. Estos aspectos, serán objeto de estudio y reflexión en la siguiente sección.

\section{Cobertura periodística: agenda político-diplomática y agenda económica}

Esta sección se focalizará en el análisis de la cobertura periodística de los principales medios gráficos de tirada nacional de Argentina (La Nación, Página/12 y Clarín) así como los Comunicados de Prensa de Cancillería relativos a la Cuestión Malvinas en el período 2011-

\footnotetext{
${ }^{4}$ La Ley $\mathrm{N}^{\circ} 26.915$, sancionada el 27 de diciembre de 2013, modifico la ley $\mathrm{N}^{\circ} 26.659$.

${ }^{5}$ La Resolución N¹94/2013 modificó la anterior $N^{\circ}$ 407/2007.
} 
2015, donde se fue testigo de un escenario complejo en materia internacional, particularmente respecto a la disputa por el archipiélago.

Para la realización de este trabajo se ha tenido en cuenta: la relación entre la frecuencia de aparición de la Cuestión Malvinas, los temas más relevantes relacionados a dicha cuestión y su vinculación con los actores en esta problemática.

De acuerdo a los datos recabados, se observa que el tópico con mayor número de apariciones $^{6}$ (86), fue el reclamo de soberanía sobre las Islas Malvinas, expresado por funcionarios argentinos en distintos contextos tanto nacionales como internacionales. Asimismo, se evidencia una serie de temas asociados a la cuestión de fondo -que es la soberanía-, que aparecieron en menor medida $^{7}$ (57), y entre los que se destacan: los respaldos internacionales provenientes de organismos regionales y extra regionales a dicha causa, y las denuncias del gobierno argentino contra su par inglés por la militarización de las islas y del Atlántico Sur. Las temáticas mencionadas registraron mayor número de aparición durante el período estudiado en torno a la Cuestión Malvinas. Además, pero en menor medida, los medios reflejaron las noticias sobre las actividades económicas como las relacionadas a la exploración y explotación del petróleo y el gas en la zona circundante a las islas, y la actividades pesqueras. En último lugar aparecieron las noticias que muestran las visitas de los familiares de los excombatientes a Darwin, y la realización del referéndum por parte de los habitantes de las Islas Malvinas.

Otro aspecto bajo estudio lo constituyen los "actores" para cuyo análisis se utiliza la clasificación de Mansbach (Barbé, 2007:284), que diferencia entre actores públicos y privados. En cuanto a la frecuencia, los que aparecieron en mayor medida fueron los actores públicos ${ }^{8}$ y los protagonistas fueron los Estados, los representantes oficiales de cada uno de ellos. En el caso argentino, las mayores frecuencias registradas recaen sobre la presidenta, el canciller, el titular de la Secretaría de Asuntos relativos a la Cuestión Malvinas, el ministro de Defensa argentino y la embajadora argentina en Londres. Ellos figuran entre los importantes exponentes de los reclamos argentinos. En menor medida observamos la aparición de las organizaciones de veteranos y familiares de ex combatientes de Malvinas.

Desde la parte británica se detecta que tuvieron mayor aparición los funcionarios oficiales como el primer ministro, el ministro de Relaciones Exteriores para América Latina, el gobernador británico designado para las Islas Malvinas y los representantes de la Asamblea de los habitantes de las islas.

En cuanto a los actores privados, fueron los que se registraron en menor medida ${ }^{9}(38), \mathrm{y}$ los más destacados fueron las empresas petroleras, a través de las declaraciones de sus representantes legales, que adquirieron las licencias para desempeñar actividades económicas en la zona bajo disputa, como también las compañías pesqueras que operan en las aguas del Atlántico Sur.

\section{Agenda político-diplomática}

Este apartado prestará especial atención a las acciones político-diplomáticas desarrolladas por la Argentina en relación a la recuperación de la soberanía sobre las Islas Malvinas en los diferentes ámbitos nacionales e internacionales, y a las denuncias contra el Reino Unido en los diferentes terrenos.

Como pudo evidenciarse anteriormente, el tema de la disputa por la soberanía entre Argentina y Reino Unido sobre las Islas Malvinas fue una de las temáticas de mayor aparición en el período bajo análisis en el presente trabajo.

\footnotetext{
${ }^{6}$ Ver Tabla TEMAS en ANEXO

${ }^{7}$ Ver Tabla TEMAS en ANEXO

${ }^{8}$ Ver Tabla ACTORES en ANEXO

${ }^{9}$ Ver TABLA ACTORES en ANEXO
} 
Vale la pena aclarar y remarcar que la discusión sobre la soberanía se encuentra estancada por la negativa del gobierno inglés a dialogar con Argentina a fin de resolver dicha cuestión en forma pacífica, tal como lo proponen las diferentes resoluciones emitidas por OI, como las Naciones Unidas y la OEA, entre otras.

A nivel gubernamental, se expresaron los reclamos por la soberanía sobre Malvinas ante la Asamblea General de las Naciones Unidas y en el Comité de Descolonización. Debe destacarse como un hecho histórico la participación de la presidenta Fernández en el Comité de Descolonización -año 2012- como la primera vez que un primer mandatario de un Estado concurrió a dicho Comité ${ }^{10}$. En su disertación presentó la Cuestión Malvinas refiriéndose a los 70 antecedentes históricos y geográficos que avalan el reclamo y solicitó al gobierno inglés que se sentara a una mesa a dialogar (Simonoff, 2014). También Fernández reiteró el no cumplimiento, por parte del Reino Unido, de las resoluciones de diversos organismos internacionales y regionales.

Es así como se destaca el rol del Poder Ejecutivo y de funcionarios de Cancillería, en la visibilización del tema Malvinas, tanto en el escenario interno como externo. Se observa que la reaparición del conflicto diplomático es uno de los ejes centrales de la administración pero, a su vez, también lo es el consolidar un espacio regional en forma de bloque que garantice el apoyo latinoamericano. Es así, como lo reflejan los medios analizados.

Todas las decisiones en materia de PE relacionadas a la Cuestión Malvinas, siguen los lineamientos generales de la PEA. Por instrucción de la presidenta, en la memoria detallada del Estado de la Nación -elaborada por la Jefatura de Gabinete de Ministros- se hace referencia precisa a dicho objetivo: "Sostener inclaudicablemente el reclamo de soberanía sobre las Islas Malvinas, Georgias del Sur y Sandwich del Sur y los espacios marítimos circundantes, respetando el modo de vida de los habitantes y conforme al Derecho Internacional". Este objetivo guarda una gran similitud con lo detallado en la Constitución Nacional de $1994^{11}$.

También en relación a ello, los medios dieron difusión a la firma de la Declaración de Ushuaia, que hace referencia a la legítima e imprescriptible soberanía argentina sobre las Islas Malvinas y reivindica las resoluciones de Naciones Unidas, instando a una solución negociada de la controversia y avalando como política de Estado el reclamo pacífico, además de advertir sobre la militarización e introducción de armas nucleares en una zona de paz.

Se observa también que, además del reclamo de la soberanía, otro de los temas que registró mayor aparición durante el período de estudio fueron los numerosos respaldos internacionales a los reclamos argentinos de soberanía sobre las Islas Malvinas. Durante la administración Fernández se implementó como estrategia la decisión de insertar la Cuestión Malvinas en todos los foros regionales y extra regionales, otorgándole visibilidad al reclamo argentino a través de una política multilateral, que presionara internacionalmente al Reino Unido a cumplir la Resolución 2065. En tal sentido Fernández llevó adelante una política que logró cosechar apoyos a nivel regional y de socios extra-bloque como Rusia, China, países africanos, India y los países árabes, entre otros. De esta manera se apuntó a transformar el reclamo argentino sobre Malvinas no sólo como causa de interés regional sino global. Los

\footnotetext{
${ }^{10}$ Es un organismo creado en 1961, encargado de monitorear e impulsar el proceso de descolonización de los territorios no autónomos bajo administración de potencias coloniales, con el propósito de poner fin al colonialismo. Actualmente, está integrado por: Antigua y Barbuda, Bolivia, Chile, China, Congo, Côte d'Ivoire, Cuba, Dominica, Ecuador, Etiopía, Federación de Rusia, Fiji, Granada, India, Indonesia, Irán (República Islámica del), Iraq, Mali, Nicaragua, Papua-Nueva Guinea, República Árabe Siria, República Unida de Tanzanía, Saint Kitts y Nevis, Santa Lucía, San Vicente y las Granadinas, Sierra Leona, TimorLeste, Túnez, Venezuela. Disponible en: www.un.org

${ }^{11}$ La primera de las disposiciones transitorias de la Constitución Nacional del año 1994, explicita que La Nación Argentina ratifica su legítima e imprescriptible soberanía sobre las islas Malvinas, Georgias del Sur y Sandwich del Sur y los espacios marítimos e insulares correspondientes, por ser parte del territorio nacional.
} 
países de América Latina respaldaron los derechos argentinos en la disputa de soberanía, a través de la aprobación de reiteradas declaraciones emanadas en el ámbito del MERCOSUR, la UNASUR, el ALBA y la CELAC.

Puntualmente, es destacable el pronunciamiento emitido por la UNASUR, donde se impugna el desarrollo de actividades unilaterales por parte de Gran Bretaña en la zona disputada, que incluyen, entre otras, la exploración y explotación de los recursos naturales renovables y no renovables de la Argentina, así como la realización de ejercicios militares.

También se reflejó la decisión de la UNASUR, el MERCOSUR y el ALBA, en apoyo al gobierno nacional, de cerrar sus puertos a todo buque que enarbole la denominada "bandera" ilegal de las islas y el intercambio de información sobre todo buque o artefacto naval con derroteros que incluyan las Islas Malvinas, Georgias del Sur y Sandwich del Sur con cargas destinadas a las actividades hidrocarburíferas y/o mineras ilegales en la plataforma continental argentina.

Además la Organización Latinoamericana de Energía (OLADE) "declaró reconocer la potestad de la República Argentina para emprender acciones legales contra las actividades de exploración y explotación de hidrocarburos no autorizadas en las Islas Malvinas, Georgias del Sur y Sandwich del Sur y los espacios marítimos circundantes" (MREyC, 2013:11).

Entre otros OI que dieron su apoyo al gobierno argentino sobre Malvinas puedes citarse a la Conferencia Permanente de Partidos Políticos de América Latina y el Caribe que reúne a más de 60 partidos de la región. La Organización hizo entrega a la embajadora argentina en México, un documento que en tal sentido afirma la soberanía argentina sobre las Islas Malvinas, Georgias del Sur, Sandwich del Sur y los espacios marítimos circundantes (2012).

Situaciones similares se dieron en la $126^{\mathrm{a}}$ Conferencia de la Unión Interparlamentaria ciudad de Kampala- en Uganda, en ocasión de la cual los representantes parlamentarios de Brasil, Uruguay, México, Chile, Cuba, Costa Rica Panamá y Ecuador suscribieron la denominada "Declaración de Ushuaia", donde se exhorta a ambos países a "reanudar las negociaciones sobre soberanía"; además, los parlamentarios latinoamericanos instaron a las partes a "abstenerse de tomar medidas que promuevan la presencia de armas nucleares en la zona de paz del Atlántico Sur".

El Parlamento cubano presentó su solidaridad con la Argentina en su reclamo por la soberanía de las Islas Malvinas y convocó a legisladores de todo el mundo a alentar la apertura de las negociaciones con Gran Bretaña.

En ocasión del primer encuentro de parlamentarios en el marco de la $18^{\text {a }}$ Edición del Foro de São Paulo (FES), se votó una declaración en apoyo a la soberanía argentina sobre las Islas del Atlántico Sur.

También la Asamblea Parlamentaria Euro-Latinoamericana (Volatero), respaldó a la Argentina por la soberanía de las Islas Malvinas.

El Parlamento de Alemania, ratificó el pedido de soberanía argentina y la búsqueda de un diálogo bilateral con Gran Bretaña.

Como se sabe, anualmente el Comité de Descolonización aprueba una resolución por la cual se insta al Reino Unido a que se siente a negociar con la Argentina para resolver en forma pacífica dicha disputa.

Del mismo modo la OEA, a través de sus repetidas resoluciones, solicita a retomar el diálogo entre Argentina y el Reino Unido.

En este sentido los medios reflejaron el respaldo regional, y también, el rechazo a las acciones unilaterales británicas por parte de los OI. Asimismo, se hicieron eco de estos lineamientos y reflejaron cada uno de los numerosos apoyos provenientes de cada presidente o jefe de Estado de países que acompañan a Argentina en esta causa, como así también, las 
resoluciones emitidas por los OI regionales como extra regionales que instaban a las partes al diálogo por la soberanía sobre los territorios y a resolver la disputa en forma pacífica.

También los medios gráficos reflejaron cada uno de los encuentros bilaterales que mantuvieron tanto la presidenta Fernández como su canciller Timerman, con los representantes de diferentes países del mundo, en los cuales expresaron su apoyo a los reclamos argentinos sobre Malvinas.

Entre ellos puede recordarse el apoyo del embajador chino en Argentina; el canciller de la República de Serbia, Vuk Jeremic; el ministro de Relaciones Exteriores de Namibia, Utoni Nujoma, que manifestó la solidaridad de su gobierno respecto a la posición argentina en la causa a Malvinas. Asimismo, el presidente uruguayo José Mujica, quien expresó -en varias oportunidades- la solidaridad de Uruguay con el reclamo argentino por las Islas del Atlántico Sur; el canciller de Costa Rica, Enrique Castillo Barrantes, quien expresó el "firme respaldo" de su país a "los legítimos derechos argentinos"; los embajadores de Arabia Saudita, Kuwait, Marruecos, y el jefe de Misión de la Liga de los Estados Árabes; el presidente Evo Morales, reafirmó el apoyo de Bolivia al reclamo argentino y el canciller de la República de Guatemala, Harold Caballeros, quien manifestó su respaldo al reclamo argentino por la soberanía de las Islas del Atlántico Sur, efectuado en foros regionales e internacionales.

Asimismo los medios indicaron los respaldos de los diferentes Grupos de Solidaridad que se formaron a partir de la Causa Malvinas. Entre ellos: el Grupo Chileno de solidaridad con Malvinas, Grupo Bolivariano de apoyo a Malvinas, el Grupo Latinoamericano y del Caribe (Grulac), donde además se rechaza la militarización del Atlántico Sur. En ocasión del I Encuentro de Grupos de Solidaridad con la Cuestión Malvinas de América del Norte (México), reunidos por el Grupo Mexicano de Solidaridad con las Islas Malvinas se respaldaron los derechos argentinos. También los 18 Grupos Europeos Pro Diálogo sobre la Cuestión Malvinas, quienes firmaron una declaración promoviendo el diálogo argentino-británico para hallar salida a la disputa de soberanía, y advirtieron sobre la militarización en la zona en conflicto.

Una de las temáticas de mayor aparición fueron las reiteradas denuncias por la militarización del Atlántico Sur, expresadas tanto por representantes/funcionarios del gobierno argentino como por los diversos OI que respaldan los reclamos nacionales.

Los medios de comunicación publicaron los pronunciamientos emitidos por el MERCOSUR, la UNASUR, el Grupo de Río, la Cumbre Iberoamericana, la Cumbre de Países Sudamericanos y Países Árabes (ASPA) y la Zona de Paz y Cooperación del Atlántico Sur (ZPCAS) respecto al incremento de militarización británica en el archipiélago.

En relación a ello, los medios reflejaron cuando la presidenta de la Nación, en el acto realizado en recordación de los veteranos de Malvinas que se efectuó en la Casa de Gobierno el 2 de abril de 2014, expresó que "la base militar nuclear de la OTAN en el Atlántico Sur (...) constituye hoy la mayor base militar existente al Sur del paralelo 50 grados Sur. Desde Malvinas se maneja todo el despliegue militar británico del Atlántico Sur, y también los sistemas de inteligencia electrónica" (Fernández, 2014).

Es menester recordar que las instalaciones militares británicas situadas en la isla Soledad poseen un centro de comando y control y una base de inteligencia electrónica que permite monitorear el tráfico naval y aéreo de la región, 800 hombres de la Real Fuerza Aérea, efectivos del ejército británico, miembros de la Royal Daly, dos pistas aéreas. A su vez la flota británica comprende: un patrullero, un buque de apoyo, un buque de investigación, una fragata, un submarino nuclear con capacidad de portar armas nucleares, un rompehielos (Bologna, 2014).

Asimismo los medios dieron cuenta del rechazo por parte del gobierno argentino frente a los ejercicios militares realizados por el gobierno inglés en el área bajo disputa. Estos actos fueron rechazados a través de los comunicados de la Cancillería argentina, como así también 
funcionarios argentinos manifestaron su desacuerdo en reiteradas ocasiones, como en el Consejo de Seguridad, en la Asamblea General y el Comité de los 24 de las Naciones Unidas.

En referencia a ello, también se hicieron eco del rechazo del gobierno argentino a la llegada a las islas del buque de guerra HSM Edinburg, y del destructor de la Marina Real británica, MS Dauntless, denunciando que la presencia de dicho submarino nuclear es contraria al Tratado para la Prohibición de las Armas Nucleares en América Latina y el Caribe (Tlatelolco, 1969).

\section{Agenda económica}

Este punto hace referencia a las noticias relacionadas a las actividades económicas ligadas a la exploración y explotación de hidrocarburos y actividades pesqueras desarrolladas en la zona de las Islas Malvinas.

Es necesario destacar que se registró un menor número de apariciones de noticias en los medios gráficos estudiados sobre las mencionadas problemáticas, en relación a las anteriormente descriptas como la soberanía y los respaldos internacionales.

Puntualmente, los medios publicaron la noticia de una petrolera británica -Falkland Oil and Gas Limited (FOGL)- que manifestó la existencia de un yacimiento de gas ubicado a 200 kilómetros de las Islas Malvinas, el cual no había satisfecho las expectativas, al decretarse un elevado costo para extraer y transportar el recurso.

También se reflejó la decisión de dos compañías -una británica y otra estadounidenseque anunciaron la contratación de una nueva plataforma petrolera para explorar en aguas de las Malvinas. La noticia detalló que "dos petroleras, una británica y otra estadounidense, contrataron una nueva plataforma petrolera para explorar en aguas de las Malvinas. Las empresas son Premier Oil y Noble Energy, y comparian la Eirik Raude, es una plataforma de exploración semisumergible de quinta generación” (Informe OPEA N³05).

En relación a dicho tema se publicó la disposición del gobierno argentino -mediante un decreto- de inhabilitar a una empresa petrolera británica -Falkland Oil and Gas (FOGL)- para realizar actividades en el país durante 20 años, y además la adquisición de dicha compañía de otra sociedad. En esa oportunidad se explicó que la "Falkland Oil and Gas (FOGL), una petrolera británica que había sido inhabilitada por un decreto argentino para realizar actividades en el país durante 20 años, comprará a Desire Petroleum para realizar una mayor exploración cerca del yacimiento Sea Lion, al norte de Malvinas (Informe OPEA N ${ }^{\circ} 279$ ).

Además, los medios reflejaron las sanciones por parte del gobierno argentino a las cinco- empresas petroleras británicas, calificadas de ilegales y "clandestinas" por la Secretaría de Energía argentina, en base al argumento de que las mismas no están inscriptas en los registros oficiales, mientras que operan, sin autorización, en la Plataforma Continental Argentina bajo soberanía de este país, violando no sólo las resoluciones de las Naciones Unidas, sino también leyes y reglamentos específicos argentinos.

En relación a esto -en junio de 2012- se aprobó la denominada ley Gaucho Rivero en la Cámara de Diputados bonaerense que prohíbe a aquellos buques de bandera inglesa que realicen actividades de exploración, perforación y explotación de recursos naturales en Malvinas, el amarre, permanencia o abastecimiento en los puertos ubicados en la provincia de Buenos Aires.

Los medios gráficos reprodujeron la noticia en Tierra del Fuego donde se negó el ingreso al puerto de Ushuaia a un crucero con bandera de una colonia inglesa. Las autoridades del puerto de Ushuaia hicieron valer la ley provincial conocida como Gaucho Rivero que señala la prohibición de "permanencia, amarre o abastecimiento u operaciones logísticas en territorio provincial de buques de bandera británica o de conveniencia".

En relación a estas temáticas aparecen como principales actores los sectores privados, ya que son las empresas de esta índole las que llevan adelante la exploración y explotación de los recursos hidrocarburíferos alrededor de las Islas Malvinas. No obstante también asoman los 
funcionarios argentinos que manifiestan su impugnación a diferentes acciones unilaterales realizadas por el Reino Unido, como son el otorgamiento de los permisos para la exploración y explotación de dichos recursos.

En lo referido a las actividades pesqueras en las aguas circundantes a las Islas Malvinas, es necesario recordar que se registraron muy pocas noticias durante el período bajo análisis.

Los medios dieron cuenta de los reclamos de los funcionarios argentinos frente al otorgamiento unilateral de licencias de pesca por parte del gobierno inglés, y las denuncias por la depredación de las especies ictícolas en las aguas circundantes a las islas.

Particularmente, se señaló que el secretario de Asuntos Relativos a las Islas Malvinas de la Cancillería argentina, Daniel Filmus, sostuvo que el principal objetivo que tiene el Reino Unido en el Atlántico Sur "es expoliar las riquezas hidrocarburíferas, minerales, pesqueras, de agua y biodiversidad, que pertenecen a 40 millones de argentinos y son parte de América Latina" (Informe OPEA N²90).

Una de las principales problemáticas de las autoridades nacionales es la pesca ilegal en la Zona Económica Exclusiva. Para paliar esta situación el gobierno nacional manifestó que los últimos años se han incrementado los controles mediante un sistema de monitoreo de las actividades pesqueras, especialmente se apuntó a la flota extranjera. Específicamente estos buques de diversas nacionalidades dedicadas a la explotación del calamar illex operan en la milla 201, pescan sin ningún tipo de control, y aún cuando se cierra la temporada isleña.

\section{Conclusiones}

Este trabajo analizó la cobertura de los periódicos argentinos La Nación, Clarín, Página/12 y los Comunicados de Prensa de Cancillería en el período 2011-2015 sobre la Cuestión Malvinas y se lo comparó con los lineamientos generales de PE del período.

La evidencia con que se ha contado permite acercar las conclusiones a la hipótesis planteada dado que, en el período bajo análisis, el tema de mayor número de aparición fue el reclamo de la soberanía sobre las Islas Malvinas, seguidos por los apoyos internacionales logrados por la posición argentina y las denuncias contra el accionar unilateral del Reino Unido que desconoce toda resolución internacional.

Otros tópicos con menor aparición son los referidos a la exploración y explotación de hidrocarburos y la explotación pesquera. También se toma en cuenta la aparición, aunque en menor medida, de noticias relacionadas a los vuelos de los ex combatientes y familiares al cementerio Darwin, en las Islas Malvinas, y el referéndum realizado por los habitantes de las islas $^{12}$.

El análisis muestra que la mayor concentración de información relativa a la controversia por la soberanía del archipiélago fue publicada durante el año 2012. Esto coincide con el inicio del segundo mandato de Cristina Fernández, donde la causa Malvinas fue una de las prioridades de su administración. También coincide con el aniversario de la guerra de Malvinas y con un escenario regional particular, en el cual Argentina adquiere mayor visibilidad en el espacio de la región.

Por lo tanto, se observó que los medios de comunicación gráfica analizados expresaron gran parte de los lineamientos de la PEA en referencia a la Cuestión Malvinas, durante el segundo gobierno de Cristina Fernández.

Analizando los resultados de la investigación, se infiere que los medios de comunicación en la Argentina muestran la reinstalación de la Cuestión Malvinas en el período

\footnotetext{
${ }^{12}$ Cabe mencionar que dicho referéndum no fue reconocido por ningún organismo regional ni por las Naciones Unidas.
} 
estudiado, aunque a veces hacen mayor hincapié en los enfrentamientos verbales y discursivos entre ambas cancillerías, y no le asignan igual importancia al estado del enfrentamiento y el estancamiento de la negociación, principalmente por la negativa del Reino Unido a negociar la cuestión de la soberanía.

Por otro lado no se muestra la importancia de los logros en el área humanitaria y no se consideran o no aparecen así los ex-combatientes de la Guerra de Malvinas como actores en el conflicto, ya que muy pocas veces son reflejados en las noticias del período.

En relación a los actores, se evidencia que los actores públicos tuvieron mayor número de apariciones con relación a los privados. Dentro de los primeros, son los Estados los que registraron mayor frecuencia de aparición. Por su parte dentro de los actores privados se identifican a las organizaciones de veteranos y familiares de ex combatientes argentinos, y a las empresas pesqueras y petroleras extranjeras que operan en las islas.

La trayectoria de la investigación deja nuevas interrogantes planteadas que estimulan nuevos análisis y reflexiones, particularmente advierte sobre la necesidad de poner el foco en el rol de los actores más relegados en la comunicación de los medios. Precisamente, son los veteranos de guerra de Malvinas, considerando que aunque tuvieron presencia durante el gobierno de Cristina Fernández, su rol en los medios no tuvo una réplica acorde a la misma.

\section{Bibliografía}

BARBÉ, E. (2007). Relaciones Internacionales, Editorial Tecnos, 3era. Edición, Madrid

BERNAL MEZA, R. (2002). Política Exterior Argentina: de Menem a De la Rúa ¿Hay una nueva política?, São Paulo em Perspectiva, 16(1), pp. 74-93

BOLOGNA, A. B. (2010). Malvinas en la política exterior argentina, en BOLOGNA, A. B. $L a$ política exterior de Cristina Fernández. Apreciaciones promediando su mandato, CERIR, UNR Editora, Rosario, pp. 243-268

BOLOGNA, A. B. (2014). Malvinas: una política activa frente al Reino Unido y a la comunidad internacional, en BOLOGNA, A. B. (comp.). La Política Exterior de Cristina Fernández al finalizar su mandato, CERIR, UNR Editora, Rosario, pp. 17-47

BUSSO, A. (2014). Los vaivenes de la política exterior argentina re-democratizada (19832013). Reflexiones sobre el impacto de los condicionantes internos", en Estudios Internacionales, Universidad de Chile, 177, pp. 9-33

BUSSO, A. (2016). Los ejes de la acción externa de Cristina Fernández: ¿cambios hacia un nuevo horizonte o cambios para consolidar el rumbo?, en Relaciones Internacionales, 50/2016

CASSESE, N. (2005), ¿Seducienco lo imposible? La estrategia argentina sobre Malvinas, Agenda Internacional, 3, pp. 82-98. Disponible en http://www.agendainternacional.com/numerosAnteriores/n3/0305.pdf

CASTILlO, I. C. (2015). Política Exterior Argentina y los medios de comunicación: la Cuestión Malvinas, Tesis Doctoral del Programa de Gestión y Políticas del Comercio Internacional, Universitat Politécnica de Valencia

CORIGLIANO, F. (2006). Híbridos teóricos y su impacto en la política exterior: el caso de los gobiernos de Néstor y Cristina Kirchner, Boletín del Instituto de Seguridad Internacionales y Asuntos Estratégicos, 47, CARI, Buenos Aires, pp. 8-10

GARCÍA LUCERO, D. (2006). La política exterior argentina durante el menemismo y su difusión en los medios de comunicación masiva, Universidad Nacional de Córdoba 
GIL, S. (1999). Las Islas Malvinas y la política exterior argentina durante los '90s: Acerca de su fundamento teórico y de la concepción de una Política de Estado, DTI No 1/1999, CARI, Buenos Aires

LASAGNA, M. (1995). Las determinantes internas de la política exterior: un tema descuidado en la teoría de la política exterior, en Estudios Internacionales, 28 (111)

MINISTERIO DE RELACIONES EXTERIORES Y CULTO (MREyC) (2013), La Comunidad Internacional y la Cuestión Malvinas, Buenos Aires

PUIG, J.C. (1984). America Latina: Políticas Exteriores Comparadas, GEL, Buenos Aires

RODRÍGUEZ DÍAZ, R. (2004). Teoría de la agenda-setting. Aplicación a la enseñanza universitaria, Observatorio Europeo de Tendencias Sociales

RUSSEL, R. (1990). Política Exterior y toma de decisiones en América Latina: aspectos comparativos y condiciones teóricas, en RUSSEL, R., Política Exterior y toma de decisiones en América Latina, GEL, Buenos Aires, pp- 255-274

SIMONOFF, A. (2009). Regularidades de la política exterior de Néstor Kirchner, en Confines, Tecnológico de Monterrey, 10, pp. 71-86

ZURITA, M.D. (2010). Las claves de la política exterior de Raúl Alfonsín, V Congreso de Relaciones Internacionales, IRI. en: http://www.iri.edu.ar/publicaciones_iri/IRI\%20COMPLETO\%20-\%20PublicacionesV05/Publicaciones/cd\%20V\%20congreso/ponencias/0\%20Zurita Las\%20claves\%20de\%20la\% 20politica\%20exterior.pdf.

\section{Sitios web}

Diario Clarín: www.clarin.com

Diario La Nación: www.lanacion.com.ar

Diario Página/12: www.pagina12.com.ar

Organización de las Naciones Unidas: www.un.org

Observatorio de Política Exterior Argentina (OPEA), UNR-GEDES, Informes de Política Exterior Argentina: https://opeargentina.wordpress.com/informes-semanales/ 


\begin{abstract}
ANEXO
De la observación general relativa a los temas más relevantes de la Política Exterior en el período 2011-2015, la Cuestión Malvinas apareció en segundo lugar de los temas de agenda, en tanto el primer lugar lo ocuparon las Relaciones Económicas Internacionales.

Los temas abordados en el texto, son reflejados de modo cuantitativo, en las siguientes tablas:

\begin{tabular}{|c|c|}
\hline \multicolumn{2}{|c|}{ TEMA } \\
\hline SOBERANÍA & ASOCIADOS \\
\hline 86 & 57 \\
\hline
\end{tabular}

\begin{tabular}{|c|c|}
\hline \multicolumn{2}{|c|}{ ACTORES } \\
\hline PÚBLICOS & PRIVADOS \\
\hline 284 & 38 \\
\hline
\end{tabular}

El tema de la soberanía es preponderante en la totalidad del período (86) en relación a los asociados (57). Asimismo, los actores públicos que aparecen son 284, en comparación con los 38 privados, a lo largo de los años analizados. El año con mayor aparición de los actores privados es el año 2012, que concuerda con el año con mayor cantidad de temas asociados. El año con mayor frecuencia del tema Cuestión Malvinas, a su vez también es el año 2012.
\end{abstract}

TABLA FRECUENCIAS

\begin{tabular}{|c|c|c|c|c|c|}
\hline AÑO & FRECUENCIAS & \multicolumn{2}{|c|}{ ACTORES } & \multicolumn{2}{c|}{ TEMAS } \\
\hline & & PÚBLICOS & PRIVADOS & SOBERANÍA & ASOCIADOS \\
\hline 2011 & 13 & 36 & 5 & 6 & 7 \\
\hline 2012 & 37 & 126 & 25 & 23 & 20 \\
\hline 2013 & 30 & 44 & 1 & 24 & 6 \\
\hline 2014 & 27 & 35 & 4 & 17 & 15 \\
\hline 2015 & 24 & 43 & 3 & 16 & 9 \\
\hline
\end{tabular}


